\title{
Sudden Onset of Swelling and Bleeding During Meningioma Surgery
}

\author{
Jeanne Teitelbaum, Douglas Fish, Line Jaques
}

Can. J. Neurol. Sci. 2008; 35: 255-259

New modalities such as hypothermia and decompressive therapy are presently being studied in the treatment of intracranial hypertension. These therapies have yet to become the standard of care. Decompressive craniotomy is reserved for local intractable oedema after severe head trauma or malignant stroke. Hypothermia is considered difficult to institute, and re-warming can lead to a rapid return of oedema. It is unclear how long hypothermia should last, and most studies have 72 hour duration or less of treatment. We present a case of severe intractable intracranial hypertension due to massive swelling and bleeding that occurred during meningioma resection. The patient was successfully treated with a prolonged and aggressive hypothermic regimen that has not been described in the literature. We discuss the management for the treatment of severe intracranial pressure with specific emphasis on prolonged hypothermia and discuss the reported incidence of bleeding dyscrasias associated with brain tumours.

\section{Case Report}

A previously well 43-year-old right-handed woman was brought to the emergency department with a two week history of increasingly severe and frequent headaches, mild memory loss, personality change and subtle gait abnormality. The neurological examination demonstrated mild psycho-motor slowing. Cranial nerves were normal, and there was no motor weakness or sensory loss. The patient was taking only vitamins and natural products at the time of presentation. The natural products were from Iran and the substances they contained could not be identified. Her blood type was A+. The CT-scan revealed a $6 \times 5 \times 6 \mathrm{~cm}$ mass lesion arising from the right frontal falx, compatible with the diagnosis of meningioma (Figure 1). She was hospitalised for surgery. The pre-operative laboratory investigations included routine biochemistry, renal function and complete blood count. All tests were normal. Coagulation studies revealed normal PTT (28.4 seconds), PT (13.2 seconds), INR (1.02) and fibrinogen $(3.0 \mathrm{~g} / \mathrm{L})$ values. Haemoglobin was 103 $\mathrm{g} / \mathrm{L}$ and platelets were $164 \times 10^{9} / \mathrm{L}$. Bleeding time was not assessed. The patient had undergone a hysterectomy two years earlier without complication.

\section{Surgical intervention}

Anaesthesia protocol: the patient was induced with Sufenta (0.3 $\mathrm{mcg} / \mathrm{kg})$, propofol (3 $\mathrm{mg} / \mathrm{kg})$ and rocuronium $(50 \mathrm{mg})$; anaesthesia was maintained with a sufenta perfusion $(0.15$ $\mathrm{mcg} / \mathrm{kg} / \mathrm{h})$, oxygen, air and desflurane. A bolus of Mannitol (0.3

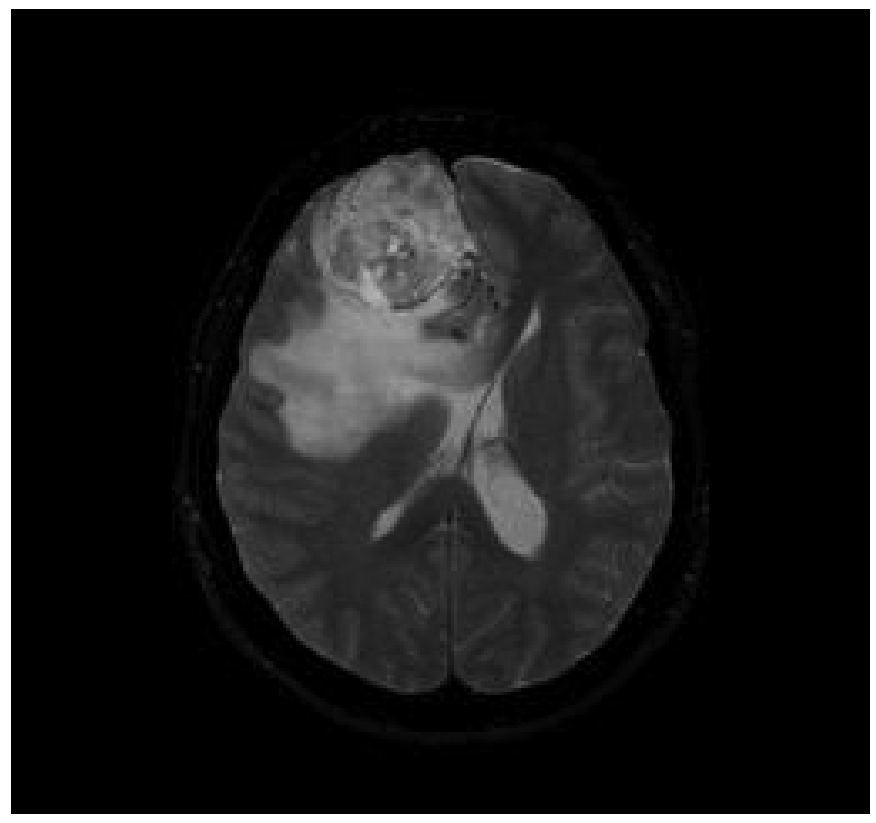

Figure 1.T2 MR of the brain, prior to surgery, showing a large mass lesion arising from the right frontal falx.

$\mathrm{mg} / \mathrm{kg}$ ) was given prior to craniotomy. Blood pressure was maintained at $90 \mathrm{~mm} \mathrm{Hg}$ systolic, and the end-tidal $\mathrm{CO}_{2}$ was kept at $35 \mathrm{~mm} \mathrm{Hg}$. During surgery, it was noted that the exposed tumour was reddish and very friable, bleeding easily when touched. The surgeons proceeded with a very slow, methodical dissection, from the posterior edge to the medial, then to the lateral edge. Blood pressure had to be maintained with an

From the Montreal Neurological Institute, McGill University; Hôpital MaisonneuveRosemont, Université de Montréal, Montreal, Quebec, Canada.

Received November 28, 2006. Final Revisions Submitted November 21, 2007. Reprint requests to: Jeanne Teitelbaum, Montreal Neurological Institute, 3801 University Avenue, Montreal, Quebec, H3A 2B4, Canada. 
ephedrine perfusion, which is unusual. About midway through the resection, the brain parenchyma began to swell. An additional bolus of Mannitol $(0.5 \mathrm{~g} / \mathrm{kg})$ was administered and end-tidal $\mathrm{CO}_{2}$ was brought to $30 \mathrm{~mm} \mathrm{Hg}$, with subsequent resolution of the oedema, and the dissection continued. Ten minutes later, the cerebral oedema recurred, and this time was accompanied by spontaneous bleeding from both the tumour bed and parts of the brain distant from the site of manipulation. An INR drawn when the bleeding began was now at 1.62 , haemoglobin was $118 \mathrm{~g} / \mathrm{dl}$, and a platelet count of $160 \mathrm{x}$ $10^{-6} /$ Liter. At the first sign of diffuse bleeding, and prior to knowing the results of the coagulation tests drawn in the operating room, the patient received in total 10 units of platelets, 10 units of cryoprecipitate, $10 \mathrm{mg}$ of vitamin $\mathrm{K}, 1 \mathrm{~g}$ of calcium chloride, 8 units of fresh frozen plasma and 20 units of vasopressin. The intra-operative treatment of cerebral oedema consisted of a total of $1500 \mathrm{ml}$ of $20 \%$ mannitol and $300 \mathrm{ml}$ of $3 \%$ hypertonic saline. End-tidal $\mathrm{CO}_{2}$ was decreased from 30 to $20 \mathrm{~mm} \mathrm{Hg}$ and the temperature was brought down from 37 to $36.3^{\circ} \mathrm{C}$. Steroids were given prior to surgery as part of the management of tumour-related vasogenic oedema. They were not given intra-operatively. Despite this ongoing therapy, bleeding and swelling continued. The surgery was aborted and it was decided to pursue further ICP management in the ICU. Closure was accomplished by suturing a large piece of dura-gard to the open edges of the patient's dura, and the bone flap was not put back. An intra-ventricular drain was inserted, and the patient was transferred to the ICU. Post operative lab results revealed a mild decrease of platelet count of 134, normal fibrinogen levels, and a bleeding time of over 20 minutes. Factor VIII and Von Willebrandt factor were both normal.

\section{ICU management}

In the ICU, therapy was initiated to manage the severe intracranial hypertension caused by the combination of oedema and haemorrhage, both of which were thought to be ongoing. Her ICP was $25-30 \mathrm{~mm} \mathrm{Hg}$ on arrival. We instituted our standard therapy for intracranial hypertension which includes hyperventilation to a $\mathrm{pCO}_{2}$ of $30 \mathrm{~mm} \mathrm{Hg}$, and the administration of mannitol $20 \% 1 \mathrm{~g} / \mathrm{Kg}$ bolus (infused in 15 minutes) followed by $0.25 \mathrm{mg} / \mathrm{Kg}$ (infused in 5 minutes) every 12 hours alternating with $250 \mathrm{cc}$ of $3 \%$ hypertonic saline also given every six hours, thus assuring the administration of hyper-osmolar agent every six hours. As end-tidal $\mathrm{CO}_{2}$ was already at $20 \mathrm{~mm} \mathrm{Hg}$ on arrival in the ICU, it was not decreased further. Extra doses of Mannitol were given when there would be sudden increases in ICP, but never more often than every four hours and there was no associated volume depletion at any time (very stable central venous pressure and strict replacement of fluid losses). The patient was receiving decadron as part of the treatment for tumour- related vasogenic oedema prior to surgery at a dose of 4 mg every six hours. After surgery, and in the context of increased oedema in the presence of persisting tumour, the patient received a $10 \mathrm{mg}$ bolus of decadron and the baseline decadron was increased to $4 \mathrm{mg}$ every four hours. As intracranial pressure was extremely high and had not responded to initial management, we began our standard protocol for refractory intracranial hypertension. This includes pharmacologic coma and hypothermia. In this case, the pharmacologic coma was induced using propofol to levels that were accompanied by burst suppression on EEG monitoring. Although the normal therapeutic goal is ICP reduction, target ICP was still not achieved despite a pattern of burst suppression on EEG. Hypothermia was being induced concomitantly using ice packs, cold IV fluids, water spray with cool air fan, and a cooling blanket. Temperature was lowered from 36.3 on arrival in ICU to 32.3 degrees Celsius in less than four hours. Our standard hypothermia protocol is based on the methods of Bernard et al in post-cardiac arres. ${ }^{1}$ Shivering occurred despite propofol and was treated with paralysis using rocuronium infusion. Norepinephrine (Levophed) infusion was required to maintain the systemic blood pressure at levels that could assure a cerebral perfusion pressure (CPP) of $60 \mathrm{~mm} \mathrm{Hg}$. We could detect no correlation between norepinephrine use and ICP. Central venous pressure was kept above $8 \mathrm{~mm} \mathrm{Hg}$ using packed red blood cells, lactate, normal saline or pentastarch as volume expanders. The use of $3 \%$ saline countered the diuretic effect of the mannitol and helped prevent dehydration. Pentaspan (pentastarch) was administered at most twice a day, to a maximum of 1 litre per day (13 ml/kg/day) from day 2 to day 11 post surgery. No pentaspan was administered prior to the operation. At that dosage, there is little danger of haemostatic compromise, and we had no evidence of any new intra or extra-cerebral haemorrhage. Mannitol was given as $250 \mathrm{cc}$ boluses (5 minute infusion) alternating with $250 \mathrm{cc}$ of $3 \%$ saline (5 minute infusion) every six hours, with extra boluses up to every two hours as needed if the ICP rose above $25 \mathrm{~mm} \mathrm{Hg}$. Serum sodium levels varied from 148 to 153 during the time of osmotic therapy. Dosing intervals and amounts of saline were modified only according to ICP and not according to sodium levels. End-tidal $\mathrm{CO} 2$ was kept between 20 and $30 \mathrm{~mm} \mathrm{Hg}$, which corresponded to an arterial pCO2 between 25 and $35 \mathrm{~mm} \mathrm{Hg}$. Over the next five days, with this regimen, ICP was kept between 15 and $20 \mathrm{~mm} \mathrm{Hg}$ most of the time, with increases treated immediately and lasting less than two minutes. Cerebral perfusion pressure was kept above $60 \mathrm{~mm}$ $\mathrm{Hg}$ at all times, and was above $70 \mathrm{~mm} \mathrm{Hg} 80 \%$ of the time. During this time, any increase in temperature by as much as $0.5 \mathrm{C}$, any manipulation or any suction would immediately raise ICP to $25 \mathrm{~mm} \mathrm{Hg}$ or more. Xylocaine was given intravenously (50 mg IV) prior to any suctioning, and both suctioning and manipulation were kept to an absolute minimum. Neither tranexamic acid nor activated factor VII were considered.

The post-operative course was complicated by a left lower lobe pneumonia that occurred seven days after surgery. We had no arrhythmias at any time. Bleeding time was prolonged during the entire time of hypothermia, but all other coagulation studies were normal and there was no evidence of bleeding. The patient received propofol at a dose of $100 \mathrm{mcg} / \mathrm{kg} / \mathrm{minute}$ for 24 hours, then $50 \mathrm{mcg} / \mathrm{kg} / \mathrm{min}(180 \mathrm{mg} / \mathrm{h}$ or $3 \mathrm{mg} / \mathrm{kg} / \mathrm{h})$ for a total of four days. This was then decreased to $25 \mathrm{mcg} / \mathrm{kg} / \mathrm{h}(1.5 \mathrm{mg} / \mathrm{h})$ for the next seven days. Lactate levels and creatinine phospho kinase (CPK) were monitored every two days while on propofol. Hepatic function was monitored every two days while hypothermic and on prolonged propofol infusion.

The first attempt at re-warming, five days following surgery, resulted in an immediate increase in ICP to $30 \mathrm{~mm} \mathrm{Hg}$ as soon as the temperature increased by $0.5^{\circ} \mathrm{C}$. Hypothermia was reinstituted and maintained for an additional seven days. Re- 


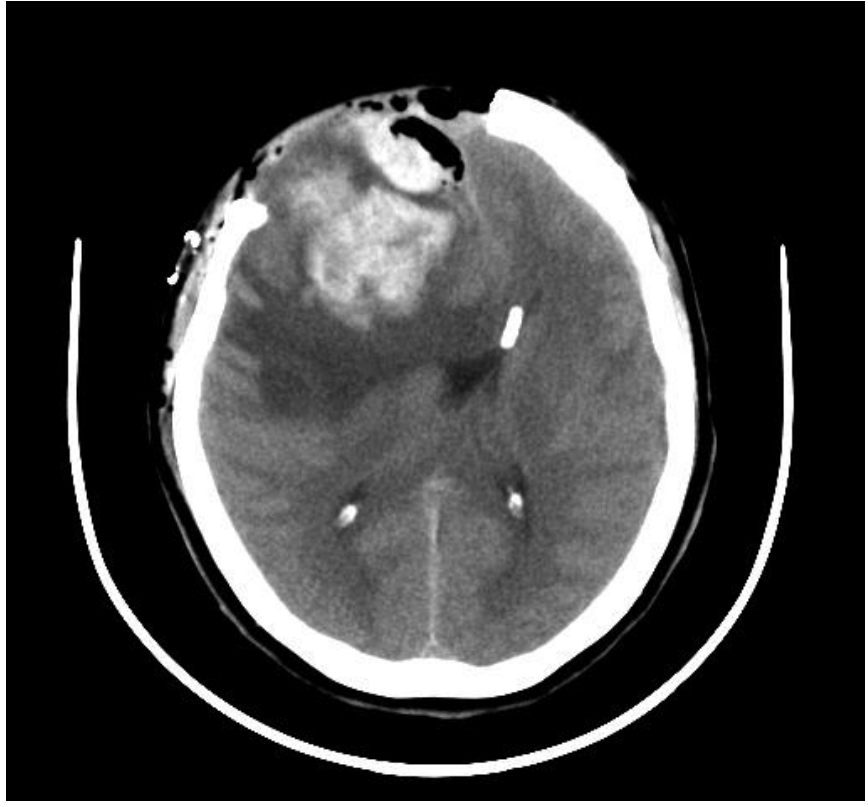

Figure 2. CT of the patient immediately after surgery.

warming was performed gradually from $32.5-33.5 \mathrm{C}$ to $34-35 \mathrm{C}$ over 24 hours, kept between 34 and $35 \mathrm{C}$ for 24 hours, then between 35 and $36^{\circ} \mathrm{C}$ for 24 hours, then increased passively to in the next 8 hours but kept always at or below $37^{\circ} \mathrm{C}$. Paralysis was necessary to stop the shivering even when poikilothermia was reached.

During these 12 days when the patient was sedated, paralysed and hypothermic, we followed the mannitol and hypertonic saline requirements, the end-tidal $\mathrm{CO}_{2}$, the response of ICP to administration of osmotic agents and changes in temperature, the CPP and serial CT (only two CT scans in the two weeks following surgery Figures 2 and 3 ) to gage the degree of response to therapy. As illustrated, haematoma volume did not increase after wound closure, and there was slow improvement of intracranial oedema and haemorrhage. The patient's oedema and haemorrhage slowly resolved, permitting the progressive withdrawal of hypothermia, propofol and paralysis. This was followed by a very slow weaning of the osmotic therapy and, finally, a progressive return of $\mathrm{pCO}_{2}$ to normal range (raised progressively over a total of three days). The patient was extubated 21 days after surgery. At that time she was fully conscious and diffusely weak because of a severe ICU myopathy (proven on EMG). Over the next three months, the myopathy resolved and the neurological examination returned to normal.

A year later, MRI showed a $4 \mathrm{~cm}$ residual mass. She underwent a second operation for complete resection of the meningioma. However, bleeding and swelling occurred very early on and the resection was aborted. INR, PTT and platelet count tested during surgery were found to be normal, and could not account for the profuse bleeding. A bleeding time could not be performed during surgery.

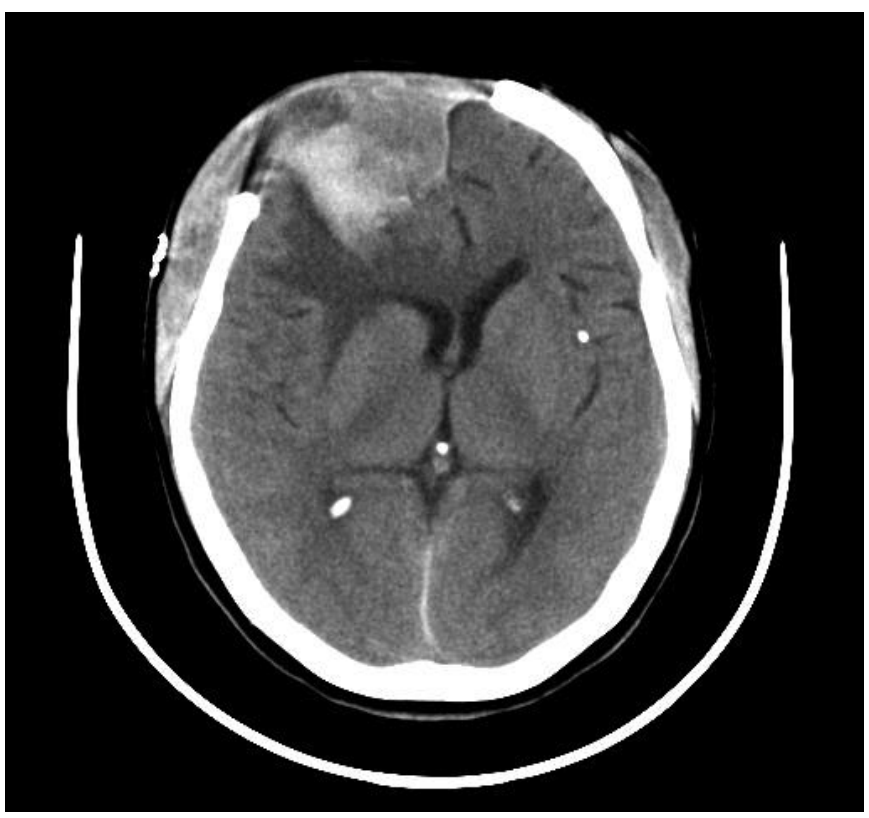

Figure 3. Head CT of the patient 9 days after surgery.

\section{DISCUSSION}

This case is unusual in that there was a sudden, severe increase in both oedema and bleeding during manipulation of an otherwise typical meningioma. The oedema was so severe that standard therapy was ineffective.

The association between coagulopathy and neoplastic diseases is well documented, and small series have been reported, describing multiple systemic coagulation abnormalities during and after craniotomy. ${ }^{2-5}$ Although most of the observed abnormalities were transient, only a minority of patients had clinically significant thrombotic or hemorrhagic events. ${ }^{6}$ Cases of repeated venous thrombosis as well as disseminated intravascular coagulation associated with meningioma have been published. ${ }^{7,8}$ Therefore, serial monitoring of systemic routine coagulation parameters may be of use during lengthy neurosurgical procedures, depending on the clinical situation.

There are previously reported cases of massive bleeding and oedema during meningioma resection. Oginashi and Tanabi, ${ }^{9}$ as well as others ${ }^{10-12}$ have reported severe and unpredictable oedema and haemorrhage occurring spontaneously in meningioma, but were unable to correlate this with either size or location. Ecosanoid release and inflammatory components have been postulated as mechanisms of oedema formation as well as other reasons such as rapid decompression of the brain after opening of the dura, unrecognised haemostatic disorders, and anaesthesia-related oedema.

In our patient, rapid decompression of a large hyper-perfused mass is an unlikely aetiology for the oedema and haemorrhage. Although midline shift was present on the preoperative MRI, the patient was asymptomatic with a normal neurological exam, and 
there was no significant swelling or bleeding when the dura was incised. It is possible, however, that the type of anaesthesia used contributed to the intra-operative swelling. The patient was induced with fentanyl and propofol, but maintained with fentanyl and desflurane. There is some evidence that autoregulation is better preserved, ICP is lower and CPP is higher if anaesthesia is maintained with propofol rather than isoflurane, ${ }^{13}$ and there is evidence that desflurane has the same effect on ICP and CPP as isoflurane. ${ }^{14}$ Despite this, it is unlikely that the aesthetic agent would be responsible for the severe oedema and bleeding that occurred, since the same oedema recurred during a second surgery using sufenta and propofol as anaesthetic agents. The most likely explanation in our patient seems to be the local

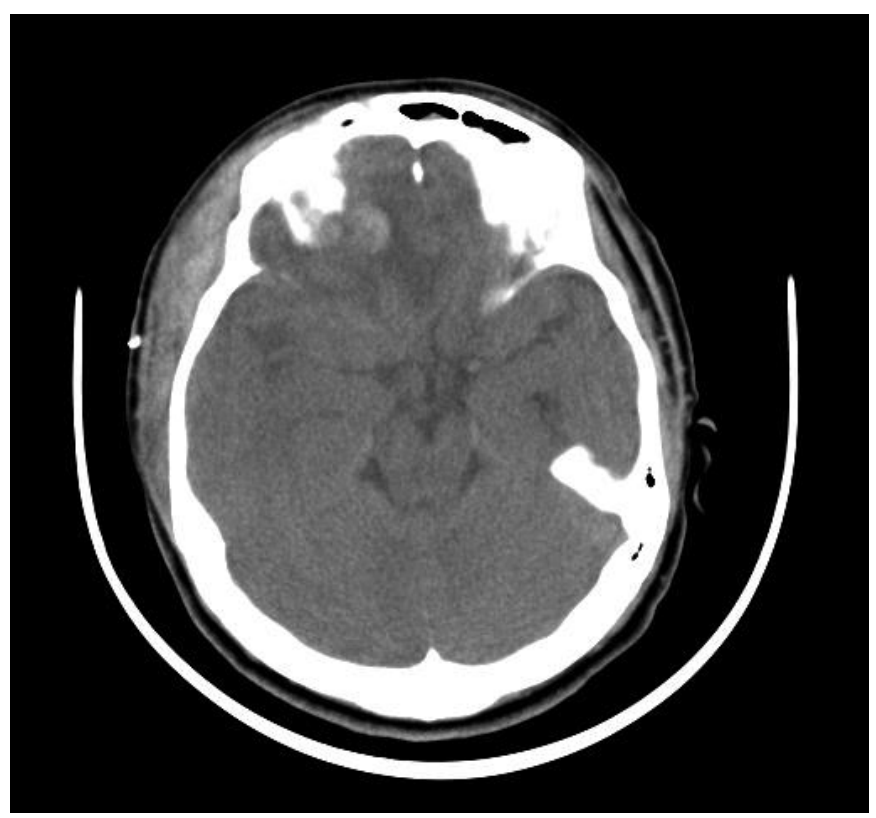

Figure 4. Head CT of the patient 15 weeks after surgery.

release of one or more factors from the tumour itself that induces the formation of oedema.

Regarding the bleeding that occurred in our patient, there was no evidence of prior coagulaopathy, personal or familial. The preoperative coagulation studies were within normal limits and the subsequent workup is incompatible with clinically silent von Willebrand's disease. Tissue dissection was not reported to be unusually difficult and it was only 11 hours from the beginning of the procedure that brisk bleeding occurred, first from the tumour and shortly thereafter from remote brain tissue. At that time, prior to fluid or blood product administration, the available routine coagulation studies showed a significant elongation of the PT, suggesting a systemic anticoagulant effect.
Again, the patient's clinical course appeared to be consistent with the release of a factor or factors locally from the tumour bed causing significant unexpected local bleeding, as well as systemic coagulopathy requiring replacement therapy with transfusion products. The temporal relationship of bleeding with tumour manipulation argue against an idiosyncratic drug induced effect. The quantity of synthetic colloid solution administered within a 24-hour-period was never greater than that recommended by the manufacturer and so it is unlikely that this therapy contributed to the observed bleeding. ${ }^{15}$ Finally, since similar local bleeding recurred early during the second operative effort a local causal effect seems most plausible.

During the active bleeding, we did not consider giving activated factor VII, aprotinin or tranexamic acid. The high intracerebral pressure had led to a very limited cerebral perfusion pressure with the danger of cerebral ischemia, and meningioma could lead to ischemic as well as hemorrhagic complications. Since the effect of these drugs could not be determined in this case, it was felt to be too risky.

The treatment of the intracranial hypertension in this case was different from accepted protocols, mainly because of the use and the duration of the hypothermia. Although moderate hypothermia is accepted as standard therapy for patients after cardiac arrest, ${ }^{1,16}$ this is not the case for stroke, and not an accepted standard of care for intracranial hypertension in general.

It is thought that lowering brain temperature may reduce injury by suppressing excitotoxin and oxygen radical formation, stabilizing cell membranes, and reducing the number of abnormal electrical depolarisations. ${ }^{17}$ Hypothermia has been studied in both animal and human models of post-traumatic oedema, malignant stroke and cardio-respiratory arrest. Improved outcome was demonstrated in two prospective, randomized, controlled trials for anoxic brain injury following resuscitation from pre-hospital cardiac arrest. ${ }^{1,16}$ Prospective, randomized, controlled trials in patients with severe head injury had more variable results, ${ }^{18}$ and although there are some preliminary clinical studies of induced hypothermia in patients with severe stroke, newborn hypoxic-ischemic encephalopathy, neurologic infection, and hepatic encephalopathy with promising results, these indications remain unproven. In all these studies looking at moderate hypothermia, the temperature was decreased to $32.5-33.5^{\circ} \mathrm{C}$ at different times after the acute event (from 2 hours, $6,8,14+/-7,22+/-9$, ) for variable lengths of time $(12,24$, 48 , and 72 hours) and with variable times for re-warming $(8,12$, 17 or 24 hours, or an increase of $0.5^{\circ} \mathrm{C}$ per 2 hours). There seems to be a beneficial effect of hypothermia on oedema, but the benefit can easily be lost during re-warming.

Hypothermia was shown to increase the incidence of nosocomial pneumonia (45\%) and hypokalemia. ${ }^{19}$ An increased incidence of cardiac arrhythmias is seen in some studies but not others. Jiang and colleagues ${ }^{20}$ compared two days of hypothermia versus five days in patients with severe traumatic brain injury. Compared with short-term mild hypothermia, longterm mild hypothermia significantly improved the outcome without significant complications. The intracranial pressure significantly rebounded after re-warming in the short-term mild hypothermia group, but not in the long-term group.

To our knowledge, this is the first reported case of acute refractory intra-cranial hypertension treated with hypothermia 
for a total of 12 days. The hypothermia seemed to have a clear effect on ICP as the ICP value seemed to correlate with the temperature. Our patient developed pneumonia that could be due to hypothermia but could also have been a complication of intubation. Bleeding time remained elevated throughout the period of hypothermia but there was no thrombocytopenia or clinical evidence of bleeding. Hypotension was more likely related to propofol than to the hypothermia itself, but this can not be proven. There were no arrhythmias during the time of hypothermia, even when the temperature transiently dipped to $31.5^{\circ} \mathrm{C}$. In general, the hypothermia was relatively easy to institute and maintain.

The severe ICU myopathy can be considered a side effect of the hypothermia in that it is likely related to the prolonged use of a paralysing agent used to stop shivering combined with type 2 fibre atrophy related to immobilisation. It recovered completely with physiotherapy.

\section{Conclusion}

Bleeding and oedema during meningioma resection has been observed and is most likely due to the release of local factors.

Prolonged hypothermia can be used in a safe manner and can help in controlling refractory increased intracranial pressure.

\section{REFERENCES}

1. Bernard SA, Gray TW, Buist MD, Jones BM, Sylvester W, Gutteridge G, et al. Treatment of comatose survivors of out-ofhospital cardiac arrest with induced hypothermia. N Engl J Med. 2002; 346:557-63.

2. Prasad KS, Sharma BS, Marwaha N, Sarode RS, Kak VK. Haemostatic derangement in patients with intracranial tumours. Br J Neurosurg. 1994; 8(6):695-702.

3. Berger MN, Ravussin P, Vielle G, Fankhauser H. Life-threatening hemorrhagic diathesis due to disseminated intravascular coagulation during elective brain tumour surgery. J Neurosurg Anesthesiol. 1995; 7(1):26-9.

4. Heesen M, Kemkes-Matthes B, Deinsberger W, Boldt J, Matthes KJ. Coagulation alterations in patients undergoing elective craniotomy. Surg Neurol. 1997; 47(1):35-8.

5. Boldt J, Papsdorf M, Rothe A, Kumle B, Piper S. Changes of the haemostatic network in critically ill patients - is there a difference between sepsis, trauma, and neurosurgery patients? Crit Care Med. 2000; 28(2):445-502.
6. Goh KY, Tsoi WC, Feng CS, Wickham N, Poon WS. Haemostatic changes during surgery for primary brain tumours. J Neurol Neurosurg Psychiatry. 1997; 63(3):334-8.

7. Heaton RB, Wright LS, Hargraves RW, Kragel P. Coagulopathy and warfarin-associated breast necrosis in a patient with a primary brain tumour. Surg Neurol. 1990 Jun; 33(6):395-9.

8. Menguy E, Fremanger P, Alibert F, Mangez JF, Roux P, Winckler C. Activation of coagulation following surgery of intracranial meningioma Presse Med. 1987; 16(12):587-8.

9. Oginashi Y, Tanabi A. A huge frontal meningioma associated with intraoperative massive bleeding and severe brain swelling--case report. J Clin Neurosci. 2001; 8(S1):54-8.

10. Jagadha V, Deck JH. Massive cerebral edema associated with meningioma. Can J Neurol Sci. 1987; 14(1):55-8.

11. Lee ST, Hsueh S. Cerebral oedema associated with meningiomas Can J Neurol Sci. 1989; 16(2):211-3.

12. Gilbert JJ, Paulseth JE, Coates RK, Malott D. Cerebral oedema associated with meningiomas. Neurosurgery. 1983; 12(6): 599-605.

13. Petersen KD, Landsfeldt U, Cold GE, Petersen C, Mau S, Hauerberg $J$, et al. Intracranial pressure and cerebral hemodynamic in patients with cerebral tumors: a randomized prospective study of patients subjected to craniotomy in propofol-fentanyl, isoflurane-fentanyl, or sevoflurane-fentanyl anesthesia. Anesthesiology. 2003; 98(2):329-36.

14. Kaye A, Kucera IJ. The comparative effects of desflurane and isoflurane on lumbar cerebrospinal fluid pressure in patients undergoing craniotomy for supratentorial tumours. Anesth Analg. 2004; 98(4):1127-32.

15. Manufacturer's insert for Pentaspan

16. The Hypothermia After Cardiac Arrest Study Group. Mild therapeutic hypothermia to improve neurologic outcome after cardiac arrest. New Eng J Med. 2002; 346(8):549-56.

17. Ginsberg MD, Busto R. Combating hyperthermia in acute stroke: a significant clinical concern. Stroke. 1998; 29: 529-34.

18. Gadkary CS, Alderson P, Signorini DF. Therapeutic hypothermia for head injury: Cochrane Database Syst Rev. 2004; Oct 18;(4):CD001048.

19. Bernard SA, Jones BM, Buist M. Experience with prolonged induced hypothermia in severe head injury. Critical Care. 1999; 3:167-72.

20. Jiang JY, Xu W, Li WP, Gao GY, Bao YH, Liang YM. Effect of long-term mild hypothermia or short-term mild hypothermia on outcome of patients with severe traumatic brain injury. J Cereb Blood Flow Metab. 2006; 26(6):771-6. 